\title{
Condensation Reactions of Phenolic Resins. VI. Dependence of the Molecular Association of 2,4,6-Trihydroxymethylphenol on the Concentration in an Aqueous Alkaline Medium
}

\author{
Naruyuki Kamo, Tetsuo Kondo, Mitsuhiro Morita \\ Graduate School of Bioresource and Bioenvironmental Sciences, Kyushu University, 6-10-1 Hakozaki, Higashi-Ku, \\ Fukuoka 812-8581, Japan
}

Received 19 September 2005; accepted 30 March 2006

DOI 10.1002/app.24471

Published online in Wiley InterScience (www.interscience.wiley.com).

\begin{abstract}
The concentration dependence of the molecular association of 2,4,6-trihydroxymethylphenol (THMP) as a model compound for phenol-formaldehyde resin was studied. ${ }^{13} \mathrm{C}$-NMR spin-lattice relaxation time $\left(T_{1}\right)$ measurements indicated that the molecular interactions were influenced by the concentration of THMP. Plots of $T_{1}$ versus the concentration revealed an inflection point around $1.2 \mathrm{~mol} / \mathrm{L}$. Molecular orbital calculations of the THMP molecules implied the following features of the molecular interactions: in the concentration range below $1.0 \mathrm{~mol} / \mathrm{L}$, THMP
\end{abstract}

existed as a single molecule, and then at a higher concentration, self-association started. At concentrations higher than $1.5 \mathrm{~mol} / \mathrm{L}$, almost all the THMP molecules were bimolecularly associated, and the formation of larger clusters was initiated. (c) 2006 Wiley Periodicals, Inc. J Appl Polym Sci 103: 2849-2854, 2007

Key words: adhesives; association; computer modeling; NMR

\section{INTRODUCTION}

Alkaline phenol-formaldehyde (PF) resins, which have superior durability and lower formaldehyde emissions, are commonly used as adhesives for various structural, wood-based materials. However, PF resins require higher reaction temperatures than $130^{\circ} \mathrm{C}$ and long reaction times for curing. Although some chemicals such as sodium carbonate and propylene carbonate are employed as reaction accelerators, ${ }^{1-6} \mathrm{PF}$ resins still require curing temperatures about $10^{\circ} \mathrm{C}$ higher than those for amino resins. To further improve the preparation conditions of $\mathrm{PF}$, the curing mechanism should be elucidated.

One step of curing of PF resins is ascribed to condensation reactions of hydroxymethylphenols (HMPs). Various HMPs are included in the prepolymer reaction system that react successively and competitively. Their reactivity changes according to the kind of HMP. Therefore, a theoretical and systematic treatment of the condensation is difficult. The behavior of monomeric HMPs as model compounds is easier to study. In previous works, the kinetics of the self-condensations of 2-hydroxymethylphenol, ${ }^{7}$ 4hydroxymethylphenol, ${ }^{8}$ 2,4-dihydroxymethylphenol, and 2,4,6-trihydroxymethylphenol (THMP) ${ }^{9,10}$ were inves-

Correspondence to: M. Morita (mimorita@agr.kyushu-u.ac.jp).

Journal of Applied Polymer Science, Vol. 103, 2849-2854 (2007) C 2006 Wiley Periodicals, Inc.

\section{(WILEY}

InterScience tigated. At THMP concentrations greater than $1.5 \mathrm{~mol} /$ $\mathrm{L}$, the reaction order was 2.0, whereas at concentrations below $1.0 \mathrm{~mol} / \mathrm{L}$, the reaction order changed to values between 1.2 and 1.6.

In this study, the dependence of the ${ }^{13} \mathrm{C}-\mathrm{NMR}$ spinlattice relaxation time $\left(T_{1}\right)$ on the concentration of THMP and the reaction temperature was investigated and complemented with results from molecular orbital (MO) calculations to clarify the molecular interactions.

\section{EXPERIMENTAL}

\section{Preparation of THMP}

Phenol (2 mol), formaldehyde (6 mol), and $\mathrm{NaOH}$ $(2 \mathrm{~mol})$ as a $50 \%$ aqueous solution were mixed under cooling, and hydroxymethylation was carried out at $20^{\circ} \mathrm{C}$ for 3 days. The mixture was then poured into $5 \mathrm{~L}$ of isopropyl alcohol to precipitate the sodium salt of THMP. The precipitate was washed with isopropyl alcohol and then dispersed in a large amount of acetone. Acetic acid was added to the dispersed mixture to liberate THMP. The precipitate of sodium acetate was filtered off, and the filtrate was concentrated with a rotary evaporator. THMP crystals formed during the concentration process. The crystals (ca. $200 \mathrm{~g}$ ) were purified by repeated recrystallization from acetone. The purified crystals were analyzed by NMR and identified as pure THMP (assay $>99.9 \%)$. 


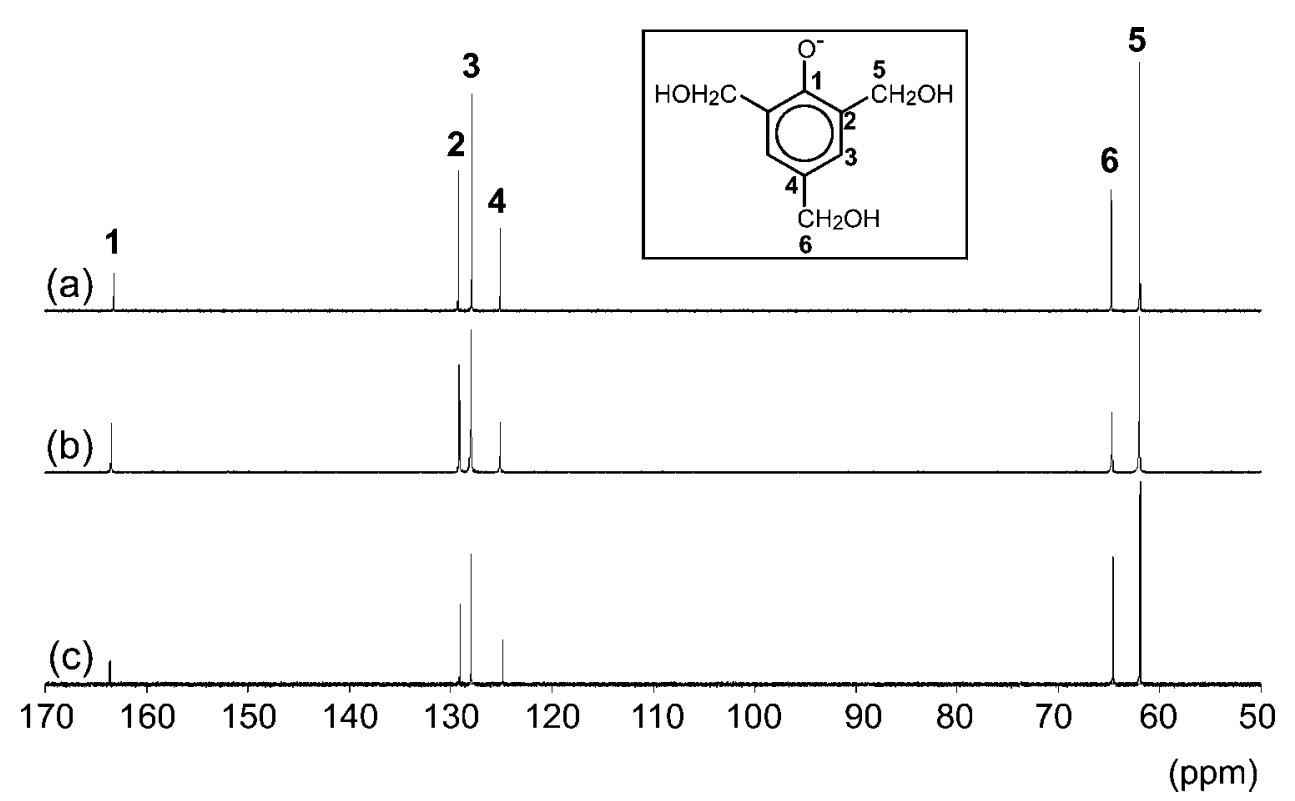

Figure $1{ }^{13} \mathrm{C}-\mathrm{NMR}$ spectra under various conditions: (a) $0.4 \mathrm{~mol} / \mathrm{L}$ and $5^{\circ} \mathrm{C}$, (b) $2.0 \mathrm{~mol} / \mathrm{L}$ and $5^{\circ} \mathrm{C}$, and (c) $0.4 \mathrm{~mol} / \mathrm{L}$ and $15^{\circ} \mathrm{C}$.

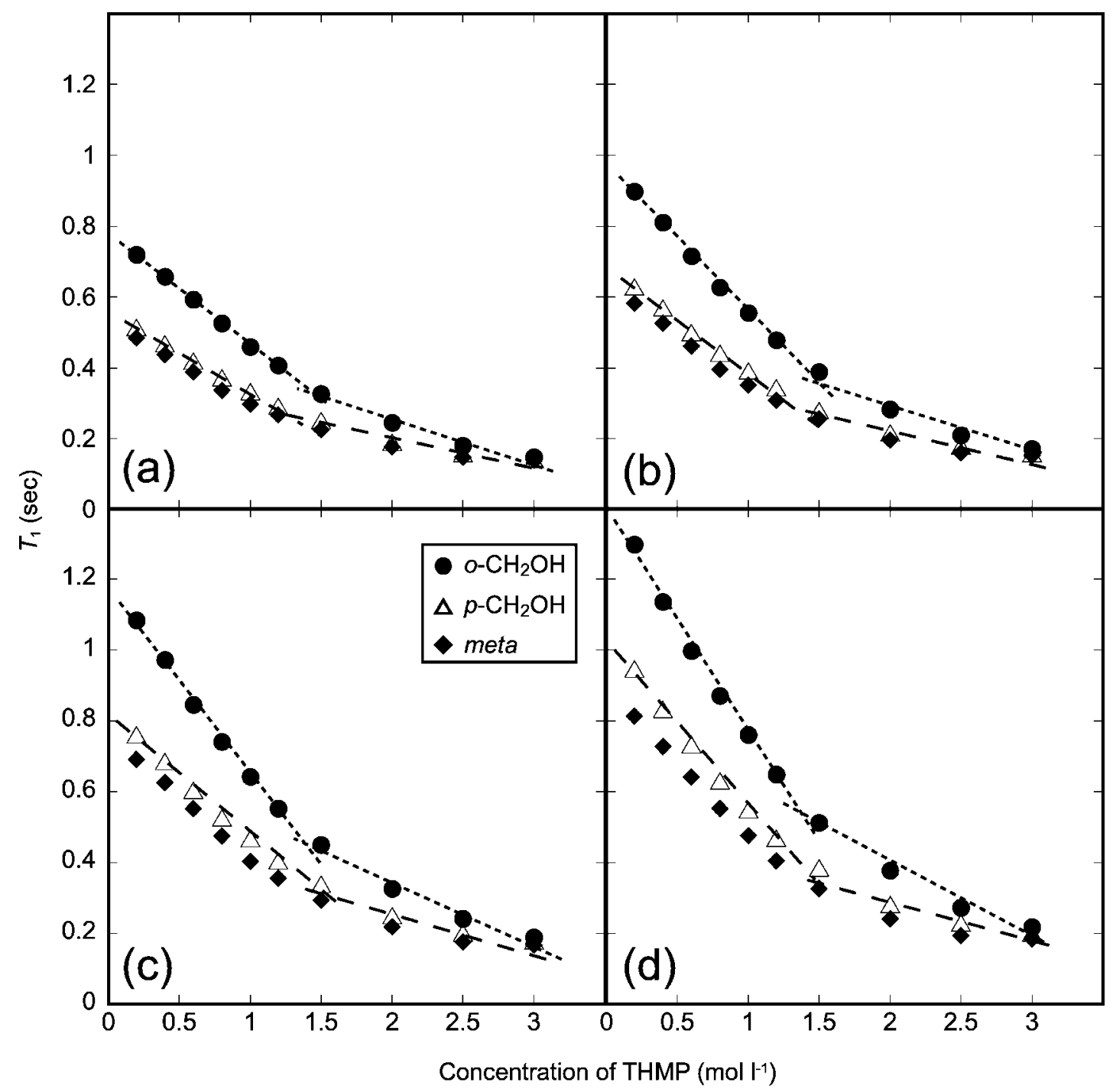

Figure 2 Dependence of $T_{1}$ on the concentration of THMP at various temperatures: (a) 5, (b) 10, (c) 15, and (d) $20^{\circ} \mathrm{C}$. Journal of Applied Polymer Science DOI 10.1002/app 


\section{${ }^{13}$ C-NMR analysis}

A JEOL JNM-AL 400 FT-NMR spectrometer (JEOL Ltd., Tokyo, Japan) with a temperature controller was used. ${ }^{13} \mathrm{C}-\mathrm{NMR}$ spectra were obtained with deuterium oxide as the solvent and sodium-3-(trimethylsilyl)propionate-2,2,3,3- $d_{4}$ as the internal standard. To make the rate of the condensation reactions negligibly slow, the measurements were carried out at 5, 10, 15 , and $20^{\circ} \mathrm{C}$. The $T_{1}$ values were determined by the inversion-recovery technique. The errors concerning these measurements were less than $5 \%$, and the reproducibility of each reaction was checked by a duplicate experiment.

\section{MO calculations}

All $\mathrm{MO}$ calculations in this work were performed with WinMOPAC (version 3.5, Fujitsu, Ltd., Tokyo, Japan). The geometries of the THMP molecule and its association were optimized with the parametric method 3 (PM3) Hamiltonian. ${ }^{11,12}$ The electron density distributions were calculated with the modified neglect of diatomic overlap (MNDO) Hamiltonian. ${ }^{13,14}$ The solvent effects of water were estimated by the conductor-like screening model (COSMO) method $^{15}$ (dielectric constant $\left.=78.4\right)$.

\section{RESULTS AND DISCUSSION}

\section{${ }^{13} \mathrm{C}$-NMR chemical shifts}

Figure 1 shows ${ }^{13} \mathrm{C}-\mathrm{NMR}$ spectra obtained under three conditions. New signals derived from condensation products or changes in the chemical shifts were not observed. Accordingly, the following $T_{1}$ measurements concerning molecular associations were not influenced by chemical reactions.

\section{Dependence of $T_{1}$ on the concentration of THMP}

Figure 2(a-d) shows the relations between $T_{1}$ and the concentration of THMP and temperature. The molecular aggregation of THMP progressed at all temperatures with increasing concentrations, resulting in solutions of higher viscosities (not shown) and a decrease in the $T_{1}$ values. All plots show an inflection point at the concentration of $1.2 \mathrm{~mol} / \mathrm{L}$. In the lower concentration region, the $T_{1}$ values of the $o$-hydroxymethyl groups $\left(T_{10}\right)$ were higher than those of both the $p$-hydroxymethyl groups $\left(T_{1 p}\right)$ and $\mathrm{C} 3$ and C5 (meta positions) of phenolic nuclei $\left(T_{1 m}\right)$. The differences between $T_{1 o}$ and $T_{1 p}$ (or $T_{1 m}$ ) decreased with an increase in the concentration of THMP. Because ${ }^{13} \mathrm{C}$ nuclei are linked to hydrogen on account of $\mathrm{CH}$ and $\mathrm{CH}_{2}$ and are relaxed predominantly by internuclear dipole-dipole interactions, the $T_{1}$ values of ${ }^{13} \mathrm{C}$ nuclei on the same structural groups can be pre- dicted to be $T_{1 o} \approx T_{1 p} .{ }^{16}$ The reason for this discrepancy between the experimental data and the prediction is thought to be due to a preferred rotation around the molecular axis passing through $C_{1}$ and $\mathrm{C}_{4}$ on the phenolic nucleus and $p$-hydroxymethyl carbon. ${ }^{16,17}$ We interpret these results as follows: THMP at lower concentrations can exist as single molecules. Moreover, THMP is assumed to self-associate progressively, and this may hinder its rotation with increasing concentrations.

\section{Dependence of $T_{1}$ on the temperature}

As shown in Figure 2(a-d), $T_{1}$ increased with increasing temperature. To quantify this dependence, the apparent activation energy $\left(E_{a}\right)$ was calculated from Arrhenius plots of $\log T_{1}$ versus the reciprocal of the temperature. $E_{a}$ decreased with an increase in the concentration of THMP (Fig. 3). At the concentration of $1.2 \mathrm{~mol} / \mathrm{L}$, the decrease was inflected, and the magnitudes of the correlations of $E_{a}$ for $o$-hydroxymethyl and $p$-hydroxymethyl groups were reversed. The inflection point at the concentration of $1.2 \mathrm{~mol} / \mathrm{L}$ agreed with that shown in Figure 2. Moreover, the inflection at this concentration is similar to the phenomenon obtained from the kinetic experiment of Kamo et al., ${ }^{9}$ indicating the change in the molecular interaction. Higher values of $E_{a}$ reflect a strong dependence of $T_{1}$ on the temperature, indicating that the hydroxymethyl group is easy to rotate. On the other hand, lower values of $E_{a}$ reflect a weak dependence of $T_{1}$ on the temperature, indicating difficulty in rotating the hydroxymethyl group. ${ }^{11}$ We interpret

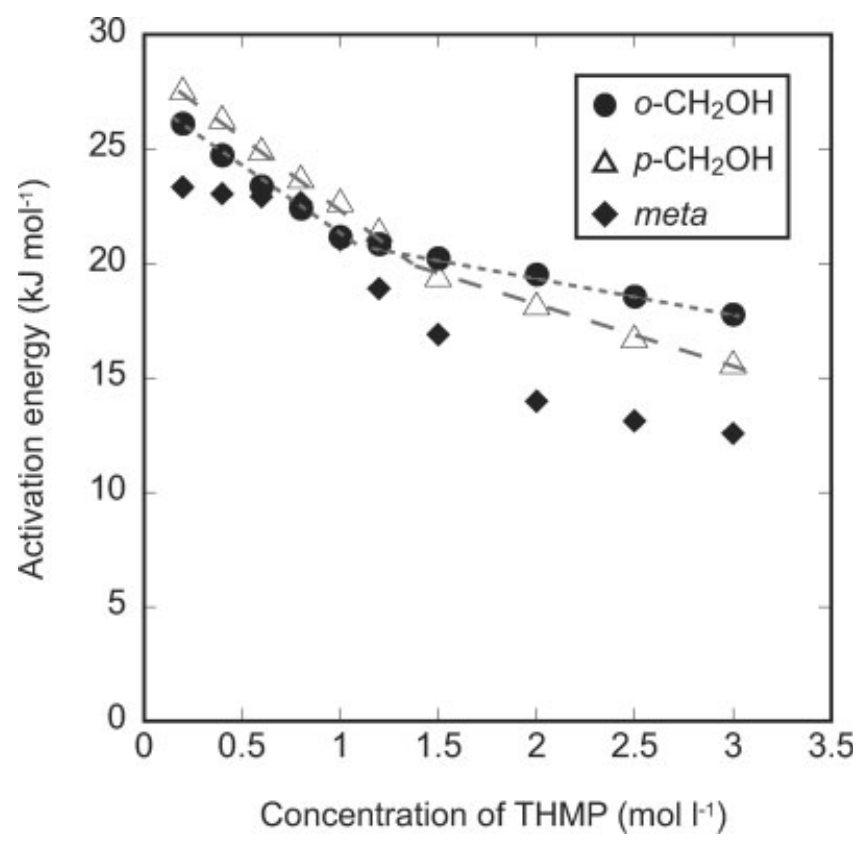

Figure 3 Dependence of $E_{a}$ on the concentration of THMP. 


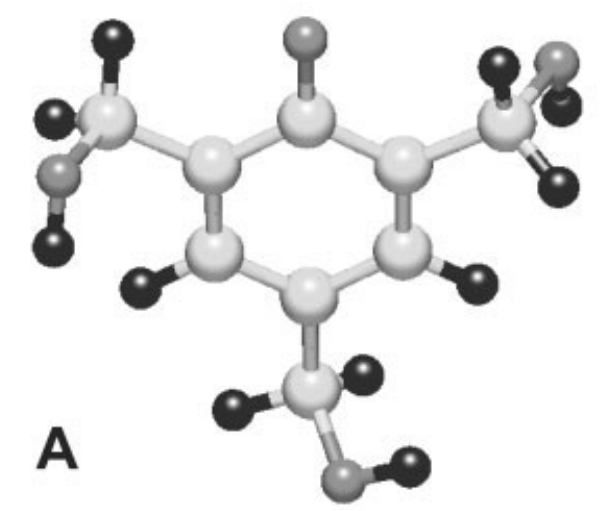

Heat of formation $=-1196.4 \mathrm{~kJ} \mathrm{~mol}^{-1}$

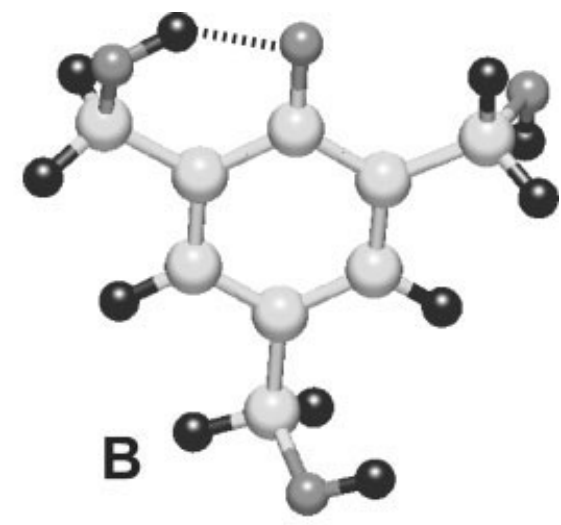

Heat of formation $=-1196.5 \mathrm{~kJ} \mathrm{~mol}^{-1}$

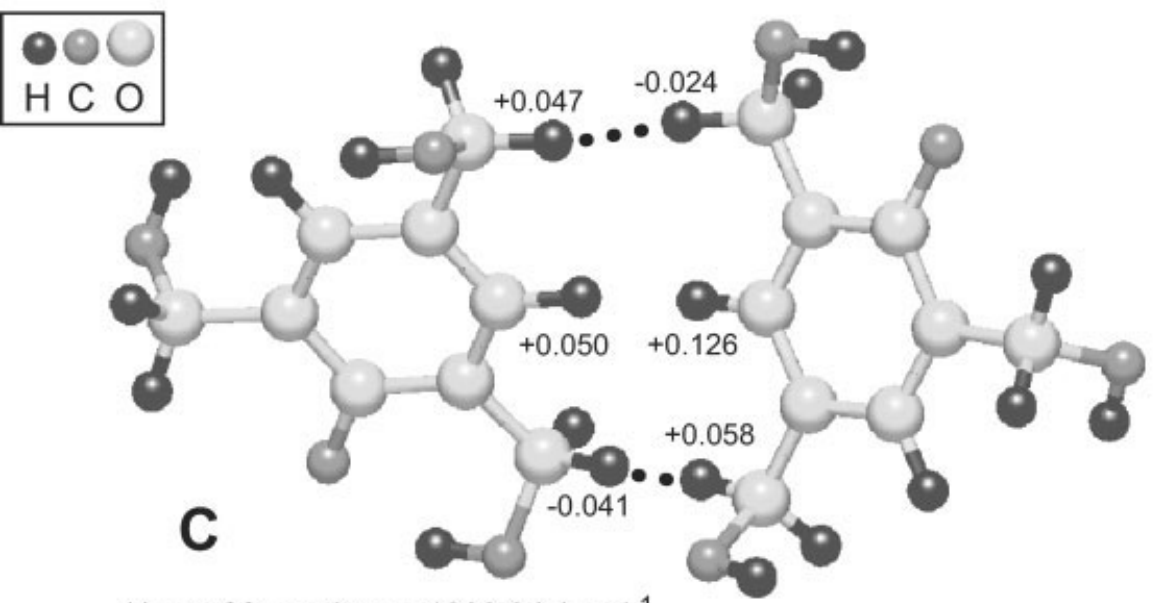

Heat of formation $=-1209.9 \mathrm{~kJ} \mathrm{~mol}^{-1}$

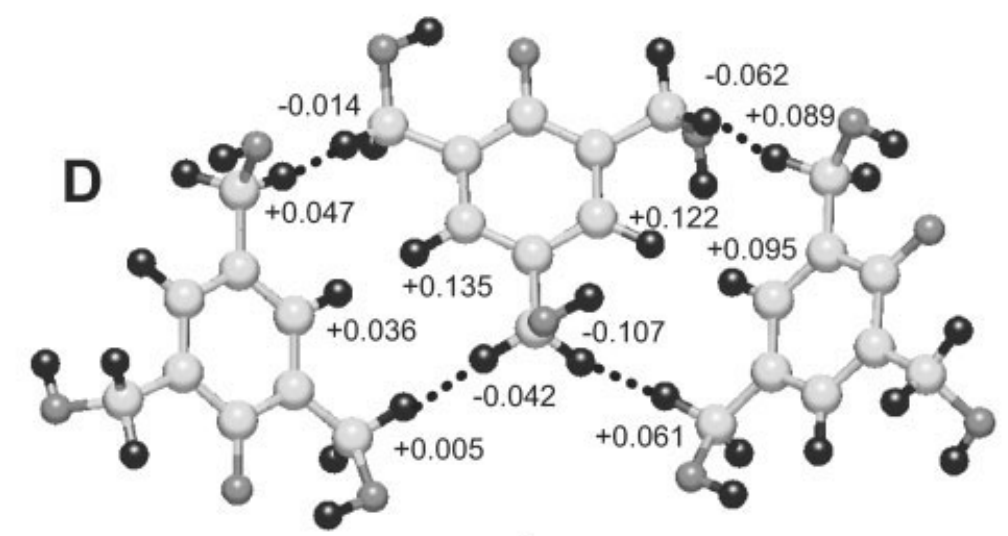

Heat of formation $=-1209.8 \mathrm{~kJ} \mathrm{~mol}^{-1}$

Figure 4 Structures of the monomeric and associated molecules of THMP and charges determined by MO calculation.

this observation by easier rotation of $p$-hydroxymethyl groups than $o$-hydroxymethyl groups. The concentration increase in THMP tends to prevent all motions of the hydroxymethyl groups. Therefore, in a higher concentration region, the motions of $p$-hydroxymethyl groups are possibly hindered more than those of $o$-hydroxymethyl groups.

We assume that in concentration regions lower than $1.0 \mathrm{~mol} / \mathrm{L}$, THMP may exist as single molecules
TABLE I

Calculated Rotation Energy of Hydroxymethyl Groups (kJ/mol)

\begin{tabular}{ccc}
\hline Structure & $o$-Hydroxymethyl group & $p$-Hydroxymethyl group \\
\hline A & 11.7 & 4.8 \\
B & 12.3 & 4.9 \\
C & 20.2 & 20.5 \\
D & 22.3 & 22.7 \\
\hline
\end{tabular}




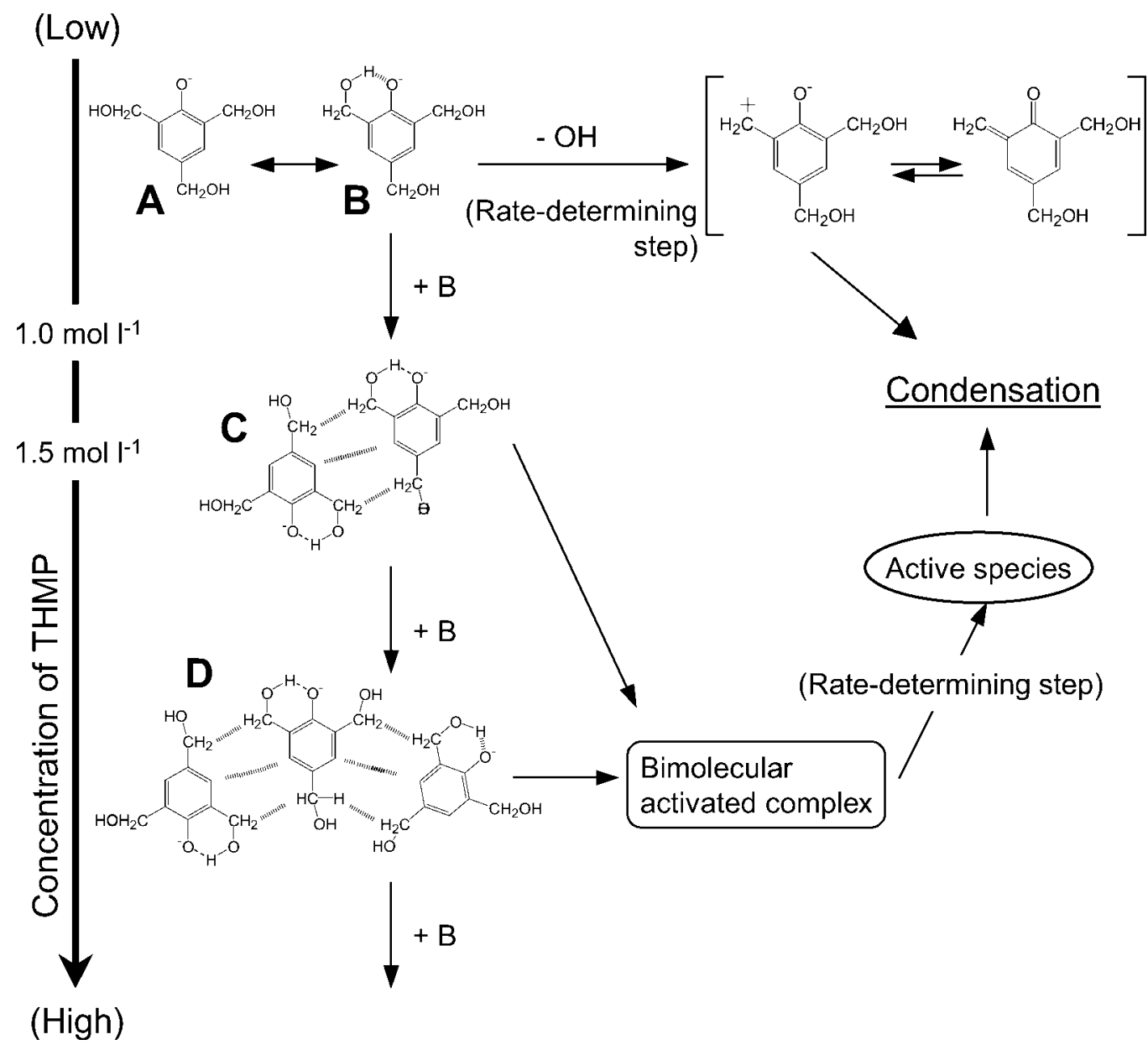

Figure 5 Possible reaction pathway of the self-condensation of THMP through molecular association in an aqueous alkaline medium.

and start to self-associate at higher concentrations. Finally, at concentrations greater than $1.5 \mathrm{~mol} / \mathrm{L}$, almost all THMP may be associated.

\section{Computational modeling of the THMP molecules and associations}

The results of the MO calculations are shown in Figure 4. Two of the most stable structures of the THMP monomer are presented. Although structure $\mathbf{A}$ has no hydrogen bond, structure $\mathbf{B}$ has an intramolecular hydrogen bond between the o-hydroxymethyl group and the phenolic hydroxyl group. The difference in the heats of formation (HOFs) of $\mathbf{A}$ and $\mathbf{B}$ is slight. Thus, they are considered to be in an equilibrium state in the solution. From the various combinations of $\mathbf{A}$ and $\mathbf{B}$, the most stable associated state of the two molecules has been determined to be structure C (Fig. 4). C comprises two molecules of $\mathbf{B}$ connected via dipole-dipole interactions. Similarly, the most stable association of the three molecules has been determined to be structure $\mathbf{D}$ (Fig. 4). D comprises three molecules of B connected via dipole-dipole interactions. To measure the degree of hindrance to the rotation of hydroxymethyl groups, we calculated the changes in HOF with a rotation of each hydroxymethyl group and defined the difference between the maximum and minimum values of HOF as the rotation energy (Table I). The rotation energies for $p$-hydroxymethyl groups of both $\mathbf{A}$ and $\mathbf{B}$ were lower than those for $o$-hydroxymethyl groups, indicating the free rotation of the former, whereas that of the latter was hindered. In the case of $\mathbf{C}$, the rotation energies for both hydroxymethyl groups were higher than those for A and B. Moreover, the rotation energy for $p$-hydroxymethyl groups was slightly higher than that for $o$-hydroxymethyl groups. When three molecules are associated, as shown in $\mathbf{D}$, the rotation energies for both hydroxymethyl groups are higher than those for the dimeric association of $\mathrm{C}$. These calculations demonstrate that hydroxymethyl groups are hindered in larger associations. In particular, the rotations of $p$-hydroxymethyl groups are affected. The results from $\mathrm{MO}$ calculations support those obtained from $T_{1}$ measurements. 


\section{CONCLUSIONS}

$T_{1}$ measurements with ${ }^{13} \mathrm{C}-\mathrm{NMR}$ and computational modeling by $\mathrm{MO}$ calculations have revealed the dependence of molecular association. In a concentration region lower than $1.0 \mathrm{~mol} / \mathrm{L}$, THMP may exist as single molecules. In the concentration range above $1.5 \mathrm{~mol} / \mathrm{L}$, almost all THMP may be bimolecularly associated and begin to form larger associations.

Considering these results together with previous kinetic works, 9,10 we assume a reaction pathway for the self-condensation of THMP (Fig. 5). One of the rate-determining steps in the low-concentration region is the production of $o$-carbocation as $o$-quinone methide through the break of the intramolecular hydrogen bond in a unimolecular reaction. Another rate-determining step in the low-concentration region is considered to be the formation of some active species via an activated complex state formed from the two molecules associated in a bimolecular reaction. In the high-concentration region, the ratedetermining step is also considered to be the formation of some active species via the some activated complex state formed from the two molecules associated in larger clusters in a bimolecular reaction.

From this reaction pathway, it can be predicted that a facilitation of the break of the intramolecular hydrogen bond followed by the formation of $o$-carbocation or the formation of the active group from
$p-\mathrm{CH}_{2} \mathrm{OH}$, especially in the lower concentration region, may accelerate the condensation reaction of HMPs. However, further experiments are needed to prove these hypotheses.

\section{References}

1. Pizzi, A.; Stephanou, A. J Appl Polym Sci 1993, 49, 2157.

2. Pizzi, A.; Stephanou, A. Holzforschung 1994, 48, 150.

3. Higuchi, M.; Tohmura, S.; Sakata, I. Mokuzai Gakkaishi 1994, 40,604 .

4. Tohmura, S.; Higuchi, M. Mokuzai Gakkaishi 1995, 41, 1109.

5. Conner, A. H.; Lorenz, L. F.; Hirth, K. C. J Appl Polym Sci 2002, 86, 3256.

6. Kamo, N.; Okamura, H.; Higuchi, M.; Morita, M. J Wood Sci 2004, 50, 236.

7. Higuchi, M.; Urakawa, T.; Morita, M. Polymer 2001, 42, 4563.

8. Higuchi, M.; Yoshimatsu, T.; Urakawa, T.; Morita, M. Polym J 2001, 33, 799.

9. Kamo, N.; Higuchi, M.; Yoshimatsu, T.; Ohara, Y.; Morita, M. J Wood Sci 2002, 48, 491.

10. Kamo, N.; Higuchi, M.; Yoshimatsu, T.; Morita, M. J Wood Sci 2004, 50, 68.

11. Stewart, J. J. P. J Comp Chem 1989, 10, 209.

12. Stewart, J. J. P. J Comp Chem 1989, 10, 221.

13. Dewar, M. J. S.; Thiel, W. J Am Chem Soc 1977, 99, 4899.

14. Dewar, M. J. S.; Thiel, W. J Am Chem Soc 1977, 99, 4907.

15. Klamt, A.; Schüümann, G. J Chem Soc Perkin Trans 2 1993, 5, 799.

16. Breitmaier, E.; Voelter, W. ${ }^{13}$ C-NMR Spectroscopy, 2nd ed.; Verlag Chemie: Weinheim: New York, 1978.

17. Sugiura, M.; Sai, T.; Takao, N.; Fujiwara, H. J Chem Soc Perkin Trans 2 1983, 7, 907. 